\title{
Heterogeneous osteoblastic activity in the right ischium of unclear etiology seen on NaF18-PET/ CT
}

\author{
Aung Zaw Win, Carina Mari Aparici \\ Dept. Radiology, Nuclear Medicine section, San Francisco Veteran Affairs Medical Center, San Francisco, USA \\ Correspondence: Aung Zaw Win. Address: 4150 Clement Street, San Francisco, CA 94121, USA. Telephone: 415-221- \\ 4810 Ext 3051. Email: Aung.Win2@va.gov.
}

Received: August 31, 2012

DOI : $10.5430 / j b g c . v 3 n 1 p 1$
Accepted: November 6, 2012 Online Published: November 19, 2012

URL: http://dx.doi.org/10.5430/jbgc.v3n1p1

\section{Abstract}

We present the case of a 54 year old white male with a history of lumbar spondylolisthesis and hip arthralgia, which prompted a hip X-ray. The X-ray showed hip arthritis as well as a sclerotic lesion in the right femur concerning for possible metastasis. He was then referred for a NaF18-PET/CT bone scan to exclude metastatic bone disease. The NaF18-PET/CT study did not show evidence of osteoblastic activity in the sclerotic right femoral lesion, but showed an incidental finding of an ill-defined intense and heterogeneous osteoblastic activity affecting the entire right ischium concerning for malignancy due to the level of activity and heterogeneity. This lesion was followed up with MRI imaging. MRI imaging has been stable and the final diagnosis is favored to be chronic avulsion injury. If a case such as ours is encountered in everyday practice, the possibility of a benign traumatic etiology should be given high consideration.

\section{Key words}

Unclear etiology, NaF18-PET/CT, Bone scan, Osteoblastic lesion

\section{I ntroduction}

We present a case of single ill-defined area of heterogeneous high osteoblastic activity in the right ischium associated with slight cortical thinning of unclear etiology seen on NaF18-PET/CT bone scan in a patient with hip pain. NaF18-PET/CT bone scan is more sensitive than Tc99m bone scan in detecting benign and malignant bone lesions ${ }^{[1]}$. This type of bone scan can produce high quality images of the skeleton due to the fact that $18 \mathrm{~F}$ has high avidity for bone which results in rapid bone uptake, creating high bone-to-background ratio in a short time. Thus, it is very suitable for skeletal exams to rule out bone pathologies ${ }^{[2]}$. The differential diagnoses of the initial findings included benign or malignant primary bone neoplasm, early Paget's disease, osteomyelitis or avulsion injury.

Bone is a common site of metastasis for carcinomas of the prostate, breast, lung, kidney, bladder, thyroid, lymphomas and sarcomas. Prostate, breast, and lung primaries account for $80 \%$ of all bone metastases. Metastatic tumors of bone are more common than primary bone tumors. Tumors usually spread to bone hematogenously. Bone metastases may be asymptomatic or may produce pain, swelling, nerve root or spinal cord compression, pathologic fracture, or myelophthisis (replacement of the marrow) ${ }^{[3]}$. 
The four most common malignant primary bone tumors are osteosarcoma, chondrosarcoma, Ewing's sarcoma, and malignant fibrous histiocytoma and they tend to be heterogeneous and very osteoblastic. Rare malignant primary bone tumors include chordoma, malignant giant cell tumor, adamantinoma, and hemangioendothelioma. Benign primary bone tumors include enchondroma, osteochondroma, chondroblastoma, chondromyxoid fibroma, osteoid osteoma, osteoblastoma, fibroma, desmoplastic fibroma, and hemangioma. Benign primary bone tumors may show some osteoblastic cortical reaction of the bone ${ }^{[3]}$.

Paget disease has a prevalence of $1 \%-2 \%$ in the United States and it affects men more commonly than women. It is usually diagnosed in patients over age 40 years and its prevalence doubles with each decade thereafter, reaching an incidence of about $10 \%$ after age $80^{[4]}$. It is usually discovered incidentally during radiology imaging or because of incidental discovery of elevation in serum alkaline phosphatase. The cause of Paget disease is unknown. However, there is often a genetic component since about 15\%-25\% of affected patients have a first-degree relative with the disease. The finding of virus-like particles in affected bones also led to the suggestion that Paget's disease may represent a virus infection of bone ${ }^{[5]}$. Paget's disease is manifested by overactive osteoclastic bone resorption followed by a compensatory increase in osteoblastic new bone formation. Paget disease is often mild and asymptomatic. Only about $27 \%$ of affected individuals are symptomatic at the time of diagnosis ${ }^{[5]}$. Bone pain may be the first symptom. It can involve just one bone (monostotic) or multiple bones (polyostotic). The skeletal sites most commonly involved are the pelvis, vertebral bodies, skull, femur, and tibia. Patients with limited monostotic involvement may have serum alkaline phosphatase levels within the normal range ${ }^{[6]}$. Paget's disease is associated with an increased risk (2\%-5\%) of malignant neoplasms in the involved bones—most often osteosarcoma, with fibrosarcoma and chondrosarcoma occurring less commonly ${ }^{[6]}$.

In the United States, osteomyelitis affects $0.1 \%-1.8 \%$ of the healthy adult population ${ }^{[7]}$. With the aging of the population and the epidemics of obesity and diabetes, this prevalence can rise. Staphylococcus aureus is the most likely bacterial pathogen ${ }^{[7]}$. Osteomyelitis of the pelvis can result from direct trauma, post-operative infection, decubitus ulcers, or hematogenous dissemination ${ }^{[7]}$. Lastly, external trauma or falling in sitting position and forceful contraction of hamstrings can cause avulsion fracture of the ischium ${ }^{[8]}$.

\section{Case presentation}

Our patient is a 54 year old white male with a history of hip arthralgia, lumbar spondylolithesis, obesity, hypertension and hyperlipidemia. He suffered a back injury from a fall about 30 years. He was complaining of persisting right hip pain, unrelieved by naproxen or tramadol. The patient stated that the pain started 2 years ago while playing handball. The pain was dull to stabbing in nature. At the beginning, he was unable to walk even a few blocks but the pain had improved gradually since then. No tenderness in the right hip area was noted in the physical exam. His primary care physician ordered an X-ray study. The X-ray image revealed an oval or flame like sclerotic lesion in the intertrochanteric portion of the right femur (see Figure 1) suspicious for metastatic disease. Consequently, the patient had a NaF-18 PET/CT whole body bone scan to rule out any bone metastases or other bone problems. The bone scan showed an incidental finding of intense heterogeneous osteoblastic activity in the right ischium associated with a rarefied cortex and areas of thin linear cortical interruption suspicious for primary bone neoplasm, Paget's disease, osteomyelitis in the right clinical setting or trauma. As a consequence he had a MRI exam 1 month later. Alkaline phosphatase (ALP) level was $42 \mathrm{U} / \mathrm{L}$ (normal 40-125 U/L). No other lab abnormalities were noted. There were no significant changes in the follow up MRI 3 months later. Orthopedic consult recommended a third MRI study in the next 3 months. 
Figure 1. X- Ray image of the right hip showing normal appearing ischium and a sclerotic lesion in the intertrochanteric portion of the right femur, adjacent to the lesser trochanter (arrow), which prompted a further evaluation by a NaF-PET/CT scan.

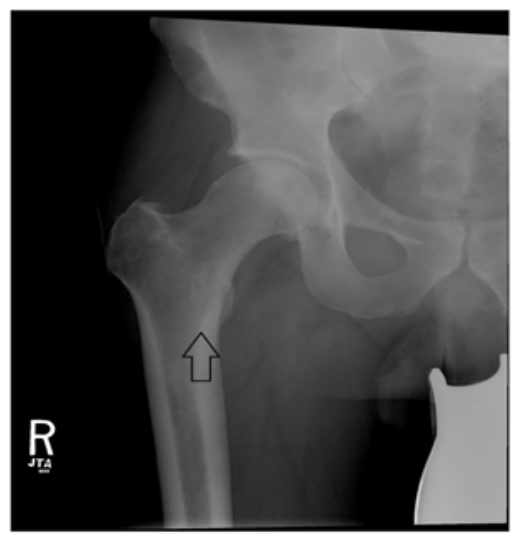

\section{Discussion}

The NaF-18 PET/CT whole body bone scan (see Figure 2) excluded widespread metastatic bone disease but the single finding had a broad differential diagnoses, including malignant primary bony lesion, benign primary bony lesion, early Paget's disease, osteomyelitis, infiltrative bone lesion and chronic avulsion injury at the hamstring attachment. Focal benign bone lesions usually show mild osteoblastic activity in the bone scans and tend to be nonspecific. In general, secondary metastasis tend to show up as multiple lesions on bone scans and solitary bony lesions are usually benign ${ }^{\text {[9] }}$. The solitary lesion on right ischium was stable on the two follow-up MRIs (see Figure 3a 3b), and the possibility of primary or secondary bone tumor was ruled out.

Figure 2. (a) whole body MIP of NaF18-PET/CT bone scan. (b,c,d) single area of intense heterogeneous, osteoblastic activity (arrows) in the right ischium associated with rarefied cortex and areas of thin linear cortical interruption in the low technique CT for attenuation correction.

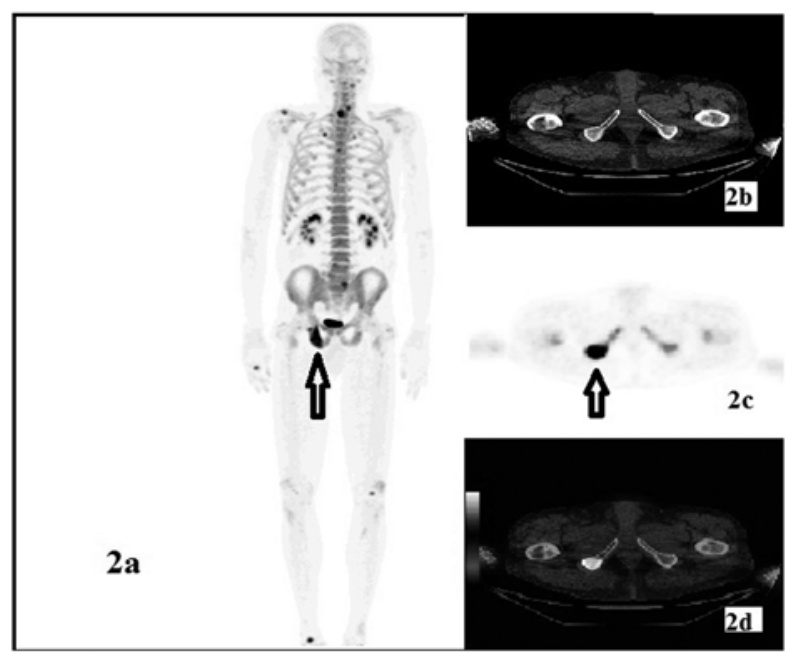

Monostotic Paget's disease involving the pelvis represents 22\% of all Paget's disease cases ${ }^{[10]}$. Elevated ALP is characteristic of Paget's disease but the rise in ALP can take decades to develop ${ }^{\text {[11] }}$. A new follow-up lab test again showed no elevation of ALP. Bone scans are helpful in detecting early Pagetic lesions even before any radiologic changes are apparent. Paget's disease tend to have very intense osteoblastic activity in metabolic imaging of the bone, usually with effect of bone expansion and even deformity. Although the intense osteoblastic activity tends to be very diffuse and homogeneous with Tc99m- MDP-bone scan imaging, it appears more patchy and heretogeneous with NaF-PET/CT imaging.

Both osteomyelitis and Paget's disease can have increased uptake on bone scans due to high perfusion to the affected areas ${ }^{[10,12]}$. Osteomyelitis was ruled out on the basis of absence of fever and elevated WBC, and no history of intervention 
in this area. Infiltrative bone lesions can have many causes and they can range from benign to malignant in nature ${ }^{[13]}$, but there are usually accompanying marrow abnormalities. The bone marrow appears normal on the two MRI studies (see Figure 3a and 3b), and infiltrative process was ruled out.

Figure 3. (a) Follow up T2 weighted MRI study one month later showed slight cortical thinning without focal mass or marrow abnormality in the right ischium. (b) Follow up MRI 3 months after the last one showed no interval changes.

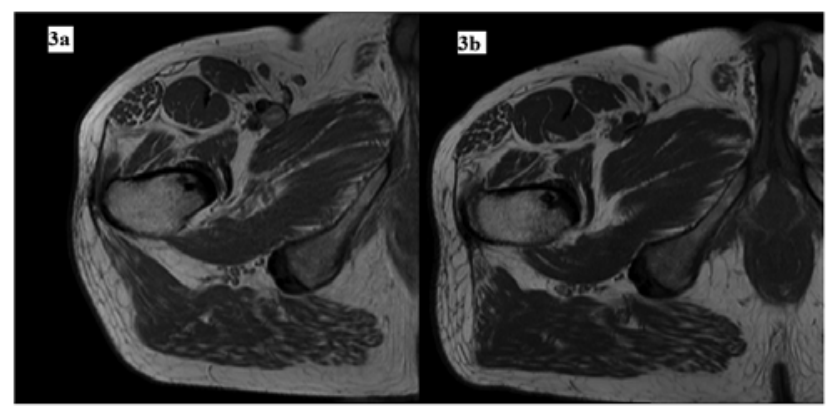

Finally, considering prior history of trauma, avulsion injury at muscle insertion was favored. Avulsion injuries of the ischium are associated with local tenderness and difficulty to ambulate ${ }^{[14]}$. Our patient had chronic pain in the right hip area and he had difficulty walking right after the injury. Chronic avulsion fractures can have prominent reactions on MRI and heterotrophic bone formations that can look like tumoral growths ${ }^{[15]}$. It is then expected to present marked osteoblastic osseous reaction in NaF-PET/CT metabolic bone imaging. Furthermore, not all avulsion injuries will have osseous displacement ${ }^{[16]}$ like the present case where only cortical thinning was observed. There may be clear signs of soft tissue injury on MRI such as a tendon tear. However, no soft tissue injury was apparent in the two MRI studies (see Figure 3a and 3b). If a case such as ours is encountered in everyday practice, the possibility of a benign traumatic etiology should be given high consideration.

\section{References}

[1] Win AZ, Aparici CM: Examining the value PSA=10ng/ml as a cutoff for predicting metastatic bone disease in NaF18 PET/CT bone scans, a pilot study. Journal of Biomedical Graphics and Computing. 2012; 2: 57-64. http://dx.doi.org/10.5430/jbgc.v2n1p57

[2] Iagaru A, Mittra E, Dick DW, et al. Prospective Evaluation of 99mTc MDP Scintigraphy, 18F NaF PET/CT, and 18F FDG PET/CT for Detection of Skeletal Metastases. Journal of Molecular Imaging and Biology. 2012; 14: 252-259. PMid:21479710 http://dx.doi.org/10.1007/s11307-011-0486-2

[3] Patel SR, Benjamin RS. Chapter. Soft Tissue and Bone Sarcomas and Bone Metastases. In: Longo DL, Fauci AS, Kasper DL, Hauser SL, Jameson JL, Loscalzo J, eds. Harrison's Principles of Internal Medicine. 18th ed. New York: McGraw-Hill; 2012: 712-724.

[4] Colina M et al. Paget's disease of bone: a review. Rheumatol Int. 2008; 28: 1069-75. PMid:18592244 http://dx.doi.org/10.1007/s00296-008-0640-6

[5] Roodman GD. Insights into the pathogenesis of Paget’s disease. Ann N Y Acad Sci. 2010; 1192: 176-80. PMid:20392234 http://dx.doi.org/10.1111/j.1749-6632.2009.05214.x

[6] Silverman SL. Paget disease of bone: therapeutic options. J Clin Rheumatol. 2008; 14: 299-305. PMid:18838910 http://dx.doi.org/10.1097/RHU.0b013e318188b1f3

[7] Ziran BH, Smith WR, Rao N. Hemipelvic amputations for recalcitrant pelvic osteomyelitis. Injury. 2008; 39: 411-418. PMid:18321513 http://dx.doi.org/10.1016/j.injury.2007.12.002

[8] Tsirikos AI et al. Transepiphyseal fracture-dislocation of the femoral neck: A case report and review of the literature. J Orthop Trauma. 2003; 17: 648. PMid:14574194 http://dx.doi.org/10.1097/00005131-200310000-00009

[9] Rosenthal DI: Radiologic diagnosis of bone metastases. Cancer. 1997; 80: 1595-1607. http://dx.doi.org/10.1002/(SICI)1097-0142(19971015)80:8+<1595::AID-CNCR10>3.0.CO;2-V

[10] Cortis K, Micallef K, Mizzi A: Imaging Paget's disease of bone-from head to toe. Clin Radiol. 2011; 66: 662-672. PMid:21524738 http://dx.doi.org/10.1016/j.crad.2010.12.016

[11] Cundy T, Reid IR: Paget's disease of bone. Clin Biochem. 2012; ahead of print. 
[12] Sethi RS, Mittal B, Bhattacharya A, et al: Tc99m MDP bone SPECT in a case of osteomyelitis of the skull. Indian J Nucl Med. 2010; 25: 25-26. PMid:20844667 http://dx.doi.org/10.4103/0972-3919.63597

[13] Steinbach LS, Suh KJ: Bone marrow edema pattern around the knee on magnetic resonance imaging excluding acute traumatic lesions. Semin Musculoskelet Radiol. 2011; 15: 208-220. PMid:21644195 http://dx.doi.org/10.1055/s-0031-1278421

[14] Porr J, Lucaciu C, Birkett S: Avulsion fractures of the pelvis - a qualitative systematic review of the literature. J Can Chiropr Assoc. 2011; 55: 247-255. PMid:22131561

[15] Akova B, Okay E: Avulsion of the Ischial Tuberosity In a young soccer player: six years follow-up. Journal of Sports Science and Medicine. 2002; 1: 27-30.

[16] Rossi F, Dragoni S: Acute avulsion fractures of the pelvis in adolescent competitive athletes: prevalence, location and sports distribution of 203 cases collected. Skeletal Radiol. 2001; 30: 127-131. PMid:11357449 http://dx.doi.org/10.1007/s002560000319 